\title{
MEDIDAS DE ADAPTACIÓN AL CAMBIO CLIMÁTICO EN UNA COMUNIDAD INDÍGENA DEL NORTE DE COLOMBIA
}

\section{MEASURES OF ADAPTATION TO CLIMATE CHANGE IN AN INDIGENOUS COMMUNITY IN NORTHERN COLOMBIA}

\author{
Andrés Galindo Montero ${ }^{1}$, Jhonny Pérez Montiel $^{2}$, Roberto Rojano Alvarado ${ }^{3}$
}

\begin{abstract}
${ }^{1}$ Doctor en Ingeniería Ambiental, Facultad de Ingeniería, Grupo GISA. Universidad de La Guajira, Km 5 vía a Maicao, Riohacha - La Guajira, Colombia, e-mail: agalindo@uniguajira.edu.co; ${ }^{2}$ Doctor en Ingeniería Ambiental, Facultad de Ingeniería, Grupo GISA. Universidad de La Guajira, Km 5 vía a Maicao, Riohacha - La Guajira, Colombia, e-mail: jpemon@uniguajira. edu.co; ${ }^{3}$ M.Sc. en Ingeniería Ambiental, Facultad de Ingeniería, Grupo GISA. Universidad de La Guajira, Km 5 vía a Maicao, Riohacha - La Guajira, Colombia, e-mail: rrojano@uniguajira.edu.co
\end{abstract}

Rev. U.D.C.A Act. \& Div. Cient. 20(1): 187-197, Enero- Junio, 2017

\section{RESUMEN}

La alta escasez hídrica que afecta las poblaciones indígenas del departamento de La Guajira, al norte de Colombia, las hace vulnerables al cambio climático y amenaza su subsistencia. En esta investigación, se implementó un modelo de adaptación al cambio climático en una comunidad indígena, integrada por 23 familias y 120 niños, de una institución educativa. Se desarrolló la metodología de Adaptación basada en Comunidades (AbC) y la evaluación rural participativa en factores claves, como hábitos higiénicos, uso y aprovechamiento del agua y subsistencia alimentaria, donde se evaluó una parcela demostrativa, con distintos sistemas de riego. Se evaluó la calidad del agua en los sistemas de suministro y de distribución, mediante el Índice de Riesgo de la Calidad del Agua para Consumo Humano (IRCA). Se instaló un sistema fotovoltaico híbrido de bombeo solar y molino de viento. Los resultados mostraron que un $88 \%$ de la comunidad consume menos de 50 L/persona*día, cantidad menor a la recomendada por la OMS (50 a $100 \mathrm{~L} /$ persona*día), para satisfacer las necesidades vitales. El IRCA registró valores de 0,0 , excepto en una muestra tomada en el sitio de acopio, con valor de 7,21, debido a la presencia de coliformes totales. El sistema de riego por goteo solar fue más eficiente, permitiendo el ahorro de agua y el sistema fotovoltaico fue viable para el abastecimiento del recurso hídrico. La comunidad, se apropió de la tecnología, conscientes de la incidencia del cambio climático y la necesidad de adoptar medidas para su mitigación y adaptación.

Palabras clave: Cambio climático, comunidad indígena, calidad de agua, sistema fotovoltaico.

\section{SUMMARY}

The high water scarcity affecting the indigenous populations of the department of La Guajira, in northern Colombia, makes them vulnerable to climate change and threatens their livelihoods. Through this research, a climate change adaptation model was implemented in an indigenous community composed of 23 families and 120 children from an educational institution. The methodology of community-based adaptation $(\mathrm{AbC})$ and participatory rural assessment were developed on key factors such as hygienic habits, water use and exploitation, and food subsistence, where a demonstration plot was evaluated with different irrigation systems. Water quality was evaluated in the supply and distribution systems through the Risk Index of Water for Human Consumption (IRCA). A solar photovoltaic system was installed for solar pumping and windmill. The results showed that $88 \%$ of the community consumes less than $50 \mathrm{~L} /$ person*day, a lower amount than that recommended by the WHO (50 to 100 L/person*day), to meet the vital needs. The IRCA recorded values of 0.0 , except in a sample taken at the collection site with a value of 7.21 due to the presence of total coliforms. The solar-powered drip irrigation system was more efficient allowing water saving and the photovoltaic system was viable for the water supply. The community appropriated this technology aware of the impact of climate change and the need to take action for its mitigation and adaptation.

Key words: Climate change, indigenous community, water quality, photovoltaic system. 


\section{INTRODUCCIÓN}

El cambio climático (CC) es un fenómeno, en el cual, se altera el estado medio del clima o su variabilidad natural, durante un periodo prolongado de tiempo y sus efectos potenciales impactan, principalmente, los sectores social, económico, ambiental y de la salud (Camare \& Lane, 2015; IPCC, 2014; Verner, 2010). A nivel mundial, los impactos del CC afectan grandes territorios, viéndose afectadas, básicamente, las zonas rurales y sus habitantes (Feldt, 2011; Ulloa et al. 2008), al igual que las regiones de latitudes bajas, en particular, las zonas costeras (Petzold \& Beate, 2015).

En la actualidad, se observa con mucha preocupación el impacto de la variabilidad climática sobre los recursos hídricos y los ecosistemas de agua dulce (Zhao et al. 2016; Melkonyan, 2015). El CC genera innumerables consecuencias, que van desde inundaciones localizadas, aumento de la temperatura y deterioro de la calidad del aire y agua (Sarzynski, 2015). En América Latina y el Caribe, se experimentan efectos negativos que pueden ser atribuidos a la variabilidad del clima, que incide sobre las clases sociales menos favorecidas y vulnerables, en particular, los pueblos indígenas, que constituyen, aproximadamente, el 6,5\% de la población latinoamericana (Solís \& Salvatierra, 2013; Kronik \& Verner, 2010; Hall \& Patrinos, 2010).

En Colombia, la mayor parte de la población reside en las zonas urbanas $(76,3 \%)$. La población presenta gran diversidad étnica: el 3,4\% pertenece a 87 etnias indígenas que, para el Departamento de La Guajira, fue estimada en 417.965 habitantes, que corresponde al $44,94 \%$ de la población, de los cuales, aproximadamente 190.000, viven en zona rural (Gobernación de La Guajira, 2016). La etnia Wayuu constituye el $90 \%$ de la población indígena, ubicada en las subregiones de la Media y Alta Guajira, donde la precipitación anual no supera los $500 \mathrm{~mm} /$ año, la evapotranspiración es muy alta, superando los $1500 \mathrm{~mm} /$ año y se registran bajas condiciones de humedad. Todas estas variables, se conjugan para generar escasez hídrica, principal problemática que padecen las comunidades indígenas residentes en estos territorios (CORPOGUAJIRA, 2009).

Ante la situación anterior, se vienen sumando esfuerzos desde hace varias décadas para hacer frente a la vulnerabilidad del clima en La Guajira, pero se ha mantenido la tradición ancestral de abastecimiento de agua en casimbas y jagüeyes, mediante la construcción de obras y el desarrollo de programas de suministro de agua con albercas, con molinos de viento, con suministro de agua con carros cisterna; sin embargo, la problemática de desabastecimiento persiste, debido a la ubicación dispersa de la población Wayuu y la falta de enfoques sostenibles, que garanticen que la infrae- structura construida se mantenga en el tiempo, con participación de las comunidades beneficiarias (MAVDT, 2010).

Investigaciones recientes han evaluado los efectos del CC en comunidades indígenas, cuya subsistencia depende directamente del uso de los recursos naturales que escasean por los cambios ya ocurridos o previsibles (Feldt, 2011). Los resultados de estos estudios han involucrado estrategias de adaptabilidad, las cuales, se enfocan en la implementación de acciones de orden técnico, para optimizar el aprovechamiento del recurso hídrico (Echeverri, 2009; Kronik \& Verner, 2010; Pérez et al. 2010; Forero et al. 2014).

La comunidad beneficiada tiene por nombre Etkojo>ole, ubicada en el departamento de La Guajira y pertenece al corregimiento La Gloria, del Municipio de Manaure, al norte de Colombia, integrada por 23 familias Wayuu y una institución educativa, que alberga a 120 niños. La investigación tuvo como objetivo implementar un modelo alternativo, como medida de adaptación al CC, para mitigar la sequía, garantizando la continuidad y la calidad del recurso hídrico, capacitaciones para mejorar los hábitos higiénicos, un mejor uso y aprovechamiento del agua y el establecimiento de cultivos como medida de adaptación de la agricultura frente a la variabilidad climática, por el manejo eficiente del agua.

\section{MATERIALES Y MÉTODOS}

La comunidad indígena Etkojo>ole está ubicada a $16 \mathrm{~km}$ del Distrito de Riohacha y pertenece al corregimiento La Gloria, del Municipio de Manaure, al norte de Colombia. La metodología desarrollada en esta investigación, se denomina: Adaptación basada en Comunidades AbC (MADS, 2013). $\mathrm{La} A b C$ es una estrategia de adaptación en que las comunidades son las protagonistas definitorias en los procesos para disminuir su vulnerabilidad y aumentar su capacidad adaptativa frente a los impactos reales o esperados del cambio climático. La metodología consiste en la capacitación en temas de cambio climático y la sensibilización sobre su importancia, la identificación y el diseño de medidas de adaptación, como se muestra en la figura 1.

Como lo plantea la guía $\mathrm{AbC}$, muchos de los resultados de los talleres desarrollados en el marco de este estudio fueron relacionados con temáticas que no son efectos directos del cambio climático, sino asuntos del desarrollo en general; sin embargo, es imposible lograr una adaptación eficaz y sostenible sin tomar en cuenta estos factores y es parte del trabajo con las comunidades apoyar todos los temas identificados (MADS, 2013), por lo tanto, se incluyeron. El análisis situacional y medidas de adaptación al CC, se realizó en fases: 


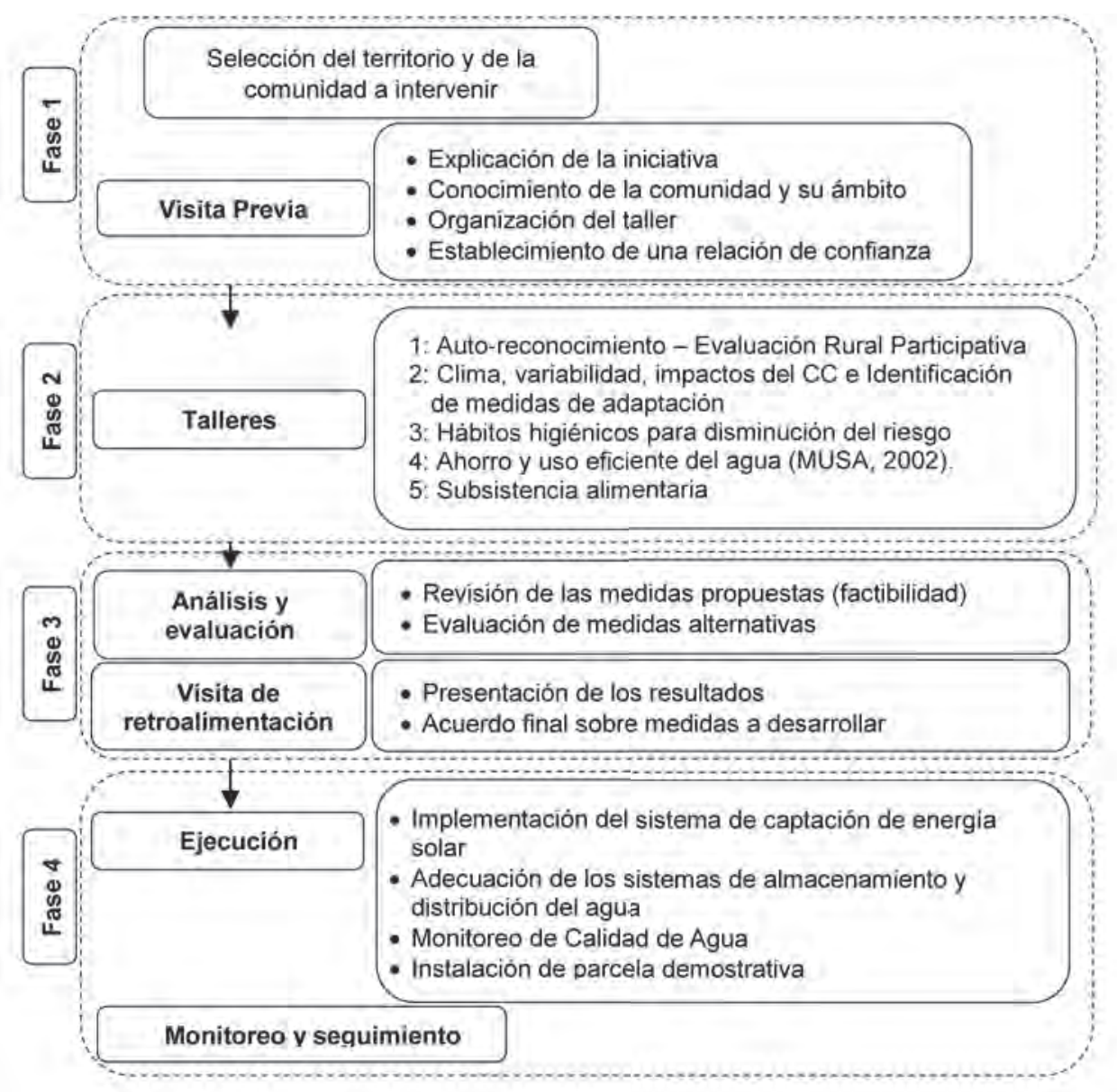

Figura 1. Diagrama metodológico, adaptado de MADS (2013).

Fase 1 -Diagnóstico preliminar-: Se realizaron citaciones y visitas a los miembros de la comunidad, a la autoridad tradicional y al líder comunitario de la etnia, para llevar a cabo un proceso de consulta y de solicitud de permisos, para la valoración del estado de la comunidad, frente a los impactos del CC en la zona.

Fase 2 -Caracterización socioambiental-: Este estudio, se basó en la metodología de Evaluación rural participativa (ERP), que comprende las actividades de caracterización, de planificación, de recolección de información y de evaluación diagnóstica (Chambers, 1994; Aubel, 2000). Mediante esta metodología, se logró observar, indagar y analizar las diferentes dinámicas sociales, culturales y económicas de los habitantes de la comunidad, en relación a CC, hábitos higiénicos, ahorro y uso eficiente del agua y subsistencia alimentaria, mediante técnicas e instrumentos de recolección de información.

En la Fase 3: Se instaló el sistema de captación de energía solar, que consistió en la adaptación de un sistema híbrido, integrado por un molino de viento ya existente y la incorporación de un sistema de bombeo fotovoltaico; para la instalación de la bomba, se realizó un video sondeo, con un equipo de grabación sumergible, para verificar las condiciones físicas de las tuberías del pozo subterráneo y establecer la ubicación de la bomba.

La Fase 4: Consistió en la adecuación de los sistemas de almacenamiento y de distribución de agua, se realizaron cuatro muestreos de calidad del agua en diferentes puntos del sistema: salida del pozo, depósito de almacenamiento y una vivienda receptora del recurso. Los muestreos, se ejecutaron antes y después de las obras de adecuación, determinando los parámetros fisicoquímicos: Temperatura, $\mathrm{pH}$, oxígeno disuelto, salinidad, conductividad, saturación de oxígeno disuelto, dureza total, turbiedad, color aparente, alcalinidad total, sólidos disueltos totales, nitrógeno amoniacal, ortofosfatos, sulfatos, cloruros, nitritos, nitratos, hierro, zinc, manganeso, calcio, coliformes fecales y coliformes totales, utilizando la metodología establecida en el Método estándar para el análisis de agua y líquidos residuales (APHA et al. 
2005). Los resultados, se compararon con la norma colombiana vigente en calidad del agua para consumo humano (MPS y MAVDT, 2007) y se realizó un análisis mediante el Índice de riesgo de la calidad del agua para consumo humano (IRCA).

Las medidas alternativas, se instalaron en el Centro Etnoeducativo La Gloria, el sistema de captación de energía solar y una parcela demostrativa de $18 \mathrm{~m}$ de ancho por $11 \mathrm{~m}$ de largo $\left(198 \mathrm{~m}^{2}\right)$, donde se evaluaron diferentes técnicas de riego: riego por goteo, usando manguera perforadas de $3 \mathrm{~mm}$ de diámetro, riego por olla porosa o de barro, regadera manual
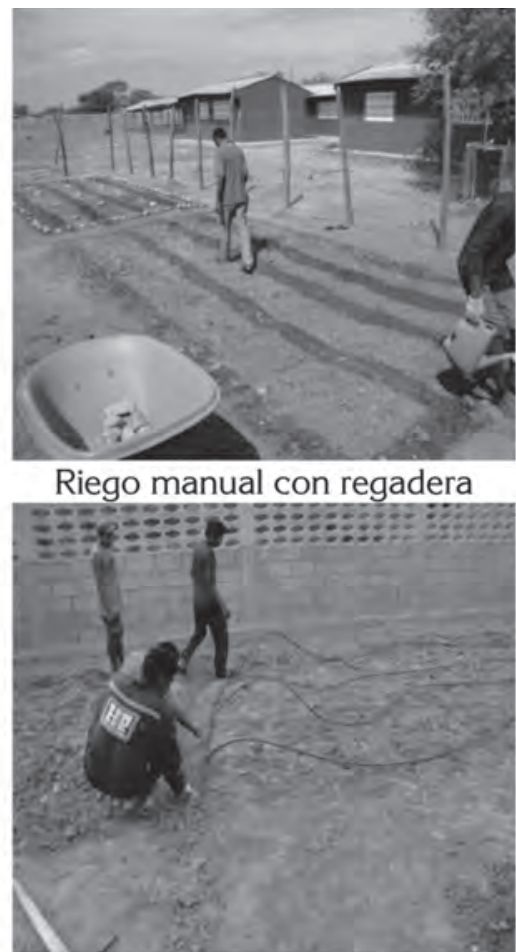

Riego por goteo con manguera y riego por goteo solar, conocido como Kondenskompressor (Figura 2), se consideraron factores como adaptación de los cultivos, demanda y control de agua (FAO, 2008). La selección del tipo de cultivo, se efectuó de acuerdo a la capacidad de adaptación a las condiciones ambientales del lugar: clima, tipo de suelo, tipos de riego, disponibilidad de agua, nutrientes y prácticas de cultivo artesanales, que se han desarrollado en la zona, seleccionando el tomate (Lycopersicum esculentum), el ají pimentón (Capsicum annuum), el frijol tinto (Phaseolus vulgaris) y el cilantro (Coriandrum sativum)
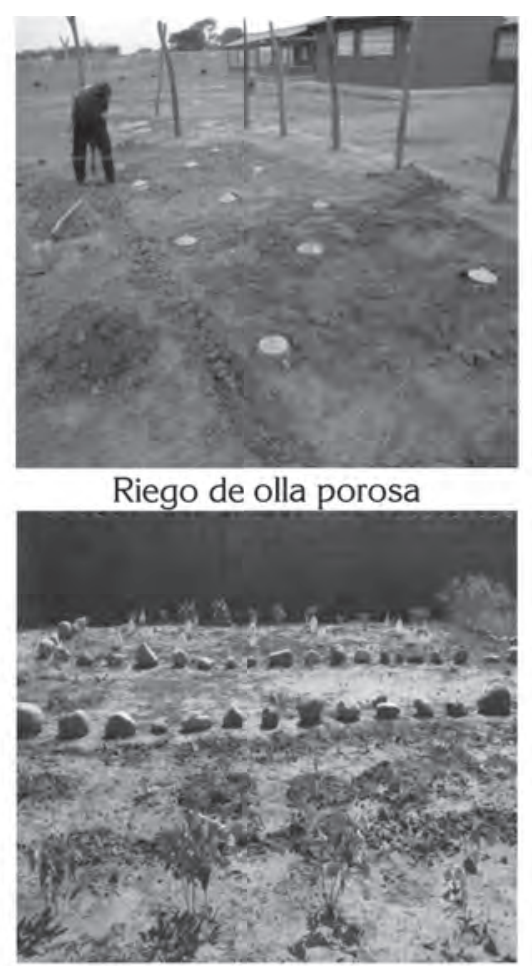

Riego por goteo solar

Kondenskompressor

Figura 2. Evaluación de los sistemas de riego.

\section{RESULTADOS Y DISCUSIÓN}

Evaluación rural participativa: La comunidad indígena Etkojo>ole fue fundada en 1971 y, actualmente, está habitada por 111 indígenas de la etnia Wayuu. En la comunidad queda ubicado el Centro Etnoeducativo La Gloria, lugar donde se concentra la mayor parte de la población infantil, perteneciente a las comunidades Etkojo>ole, Karraisira y Paranachimana, estas dos últimas aledañas a la zona de estudio, con una población de 120 niños. Los aspectos determinados mediante la ERP, se presentan la tabla 1.
La comunidad Etkojo>ole hace parte del grupo de las llamadas rancherías, pertenecientes a la zona rural dispersa del municipio de Manaure, que cuenta con 7.743 puntos poblados en dicha zona, con un promedio poblacional de más de 100 habitantes (Gobernación de La Guajira, 2016). El número promedio de personas por hogar en Manaure es de 7 y la comunidad pertenece al $49 \%$ hogares que tiene 4 o menos personas, según las cifras presentadas por el DANE (2005). 
Tabla 1. Resultados de la Evaluación Rural Participativa.

\begin{tabular}{|c|c|c|c|c|c|c|}
\hline ASPECTO & \multicolumn{6}{|c|}{ EVALUACIÓN } \\
\hline \multirow[t]{2}{*}{$\begin{array}{l}\frac{1}{0} \\
\frac{0}{0} \\
0 \\
0 \\
0\end{array}$} & \multicolumn{3}{|c|}{$\begin{array}{c}\text { Niños } \\
\text { Jóvenes } \\
\text { Mujeres } \\
\text { Hombres } \\
\text { Adultos Mayores }\end{array}$} & \multicolumn{2}{|c|}{$\begin{array}{l}\text { 0-12 años } \\
13-17 \text { años } \\
18-50 \text { años } \\
18-50 \text { años } \\
>50 \text { años }\end{array}$} & $\begin{array}{l}28 \% \\
25 \% \\
22 \% \\
19 \% \\
6 \% \\
\end{array}$ \\
\hline & \multicolumn{3}{|c|}{ \# Integrantes grupo Familiar Promedio } & & & 4 Personas \\
\hline  & \multicolumn{3}{|c|}{$\begin{array}{l}\text { Agua } \\
\text { Acueducto y Alcantarillado } \\
\text { Gas } \\
\text { Energía } \\
\text { Recolección de Residuos Sólidos }\end{array}$} & \multicolumn{3}{|c|}{$\begin{array}{c}\text { Sistema de extracción de Agua Subterránea } \\
\text { Micro acueducto - Pozos sépticos } \\
\text { No - Uso de leña } \\
\text { Si } \\
\text { No -Quema a Cielo Abierto }\end{array}$} \\
\hline \multirow{12}{*}{ 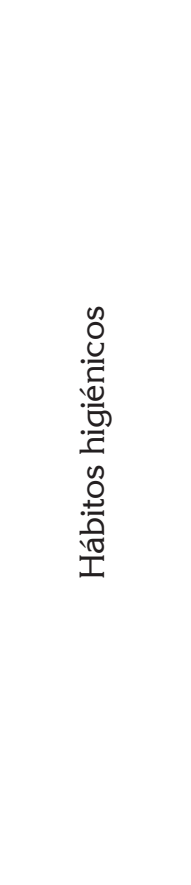 } & & & & SI & NO & $\begin{array}{l}\text { ALGUNAS } \\
\text { VECES }\end{array}$ \\
\hline & Lavado de r & Ss antes de comer & & $12 \%$ & $70 \%$ & $18 \%$ \\
\hline & Lavado de r & ss después del ba & & $29 \%$ & $65 \%$ & $6 \%$ \\
\hline & Lavado de a & ntos antes de cor & Imo & $24 \%$ & $0 \%$ & $76 \%$ \\
\hline & Hervido de & & & $0 \%$ & $100 \%$ & $0 \%$ \\
\hline & Tapado de & des de almacenam & nto de Agua & $0 \%$ & $100 \%$ & $0 \%$ \\
\hline & \multicolumn{3}{|c|}{ Almacenamiento de agua para consumo } & $\begin{array}{c}\text { Tanque Plástico } \\
88 \%\end{array}$ & $\begin{array}{c}\text { Recipiente de } \\
\text { Aluminio } \\
6 \%\end{array}$ & $\begin{array}{c}\text { Otros } \\
\text { Recipientes } \\
\text { Plásticos } \\
6 \%\end{array}$ \\
\hline & & & & 1/SEMANA & 1/MES & NO SE LAVA \\
\hline & \multicolumn{3}{|c|}{ Frecuencia de lavado de recipientes } & $18 \%$ & $6 \%$ & $76 \%$ \\
\hline & \multicolumn{3}{|c|}{ Frecuencia de lavado de baños } & $0 \%$ & $0 \%$ & $100 \%$ \\
\hline & \multicolumn{3}{|c|}{ Frecuencia de Aseo hogar } & $100 \%$ & $0 \%$ & $0 \%$ \\
\hline & \multicolumn{3}{|c|}{$\begin{array}{l}\text { Frecuencia de lavado del depósito de } \\
\text { almacenamiento colectivo }\end{array}$} & $\begin{array}{l}1 / \mathrm{MES} \\
12 \%\end{array}$ & $\begin{array}{l}1 / \mathrm{AÑO} \\
53 \%\end{array}$ & $\begin{array}{l}2 / \mathrm{AÑO} \\
35 \%\end{array}$ \\
\hline \multirow{3}{*}{ 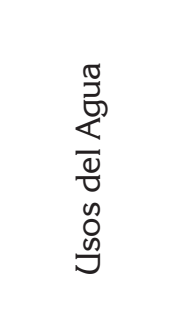 } & \multicolumn{3}{|c|}{ Control del Agua } & $\begin{array}{l}\text { Autoridades } \\
70 \%\end{array}$ & $\begin{array}{c}\text { No Sabe } \\
18 \%\end{array}$ & $\begin{array}{c}\text { Nadie } \\
12 \%\end{array}$ \\
\hline & \multicolumn{3}{|c|}{ Usos de Agua } & Doméstico & $\begin{array}{l}\text { Abrevadero de } \\
\text { Animales }\end{array}$ & Riego \\
\hline & \multicolumn{2}{|c|}{ Consumo de Agua/Persona } & $\begin{array}{l}\text { 5-15L día } \\
6 \%\end{array}$ & $\begin{array}{l}\text { 20-30L/día } \\
35 \%\end{array}$ & $\begin{array}{l}\text { 35-50L/día } \\
47 \%\end{array}$ & $\begin{array}{l}>50 \mathrm{~L} / \text { día } \\
12 \%\end{array}$ \\
\hline $\begin{array}{l}\text { Cambio } \\
\text { Climático }\end{array}$ & Percepción & $\begin{array}{l}\text { Incremento de } \\
\text { Temperatura }\end{array}$ & $\begin{array}{l}\text { Cambios en } \\
\text { Periodo de } \\
\quad \text { lluvias }\end{array}$ & $\begin{array}{l}\text { Disminución de } \\
\text { la Precipitación }\end{array}$ & $\begin{array}{c}\text { Disminución } \\
\text { Humedad de } \\
\text { Suelo }\end{array}$ & $\begin{array}{l}\text { Perdida de } \\
\text { paisaje }\end{array}$ \\
\hline \multicolumn{7}{|c|}{ / : Vez o Veces Por } \\
\hline
\end{tabular}


Dado que el agua se puede contaminar en la fuente de suministro por el ingreso de contaminantes durante la distribución del agua y dentro de la vivienda, por el uso de recipientes mal protegidos o por la manipulación insalubre del agua (RIPDA, 2003), la evaluación detectó uso inadecuado del agua, por parte de la comunidad. Además, el $88 \%$ de la población de la comunidad consume entre 5 y 50L/persona*día, lo cual, está por debajo del rango recomendado por la OMS et al. (2010), para satisfacer las necesidades vitales de higiene personal, fijado entre 50 y 100L/persona*día.

Los procesos de participación social permitieron que la comunidad, como cultura tradicional, describiera sus propias percepciones e interpretaciones sobre los cambios ambientales y climáticos de la región, hechos que se han reflejado principalmente en la disminución o ausencia del recurso hídrico, disponible para actividades domésticas, cultivos y pastoreo de ganado caprino, incrementos de la temperatura, cambios de los periodos de lluvia y sequía, por la disminución de la precipitación, reduciendo la periodicidad de las prácticas agrícolas. La pérdida de paisaje y la migración de especies biológicas, para la caza y alimentación; en térmi- nos generales, van en contra de su forma de vida tradicional, generando un desequilibrio en la organización social y aumentando los niveles de pobreza.

Sistema fotovoltaico y bomba solar de succión: La instalación del sistema fotovoltaico para la extracción del agua del pozo procedió con el montaje de 9 paneles, con 60 celdas fotovoltaicas cada uno, que generan 240 vatios de energía, para un total de 2.160 vatios/hora pico, controlados por un regulador, que gradúa la potencia de los paneles a la bomba sumergible. El video sondeo realizado en el pozo permitió ubicar la bomba, a una profundidad de $22 \mathrm{~m}$, lugar donde el contenido de sólidos fue más bajo. La bomba y la tubería del molino fueron conectadas a la tubería, que conduce el agua al tanque elevado, a través de una manguera de una 1" de diámetro; la tecnología fotovoltaica solo trabajó cuando el molino no fue suficiente para el abastecimiento o no fue posible su funcionamiento. En esta investigación, se activó el funcionamiento de un tanque elevado, que recibía el agua de pozo y alimentaba la alberca a nivel del suelo. El sistema instalado generó un caudal de $0,71 \mathrm{~L} / \mathrm{s}$. La figura 3 muestra el sistema fotovoltaico.
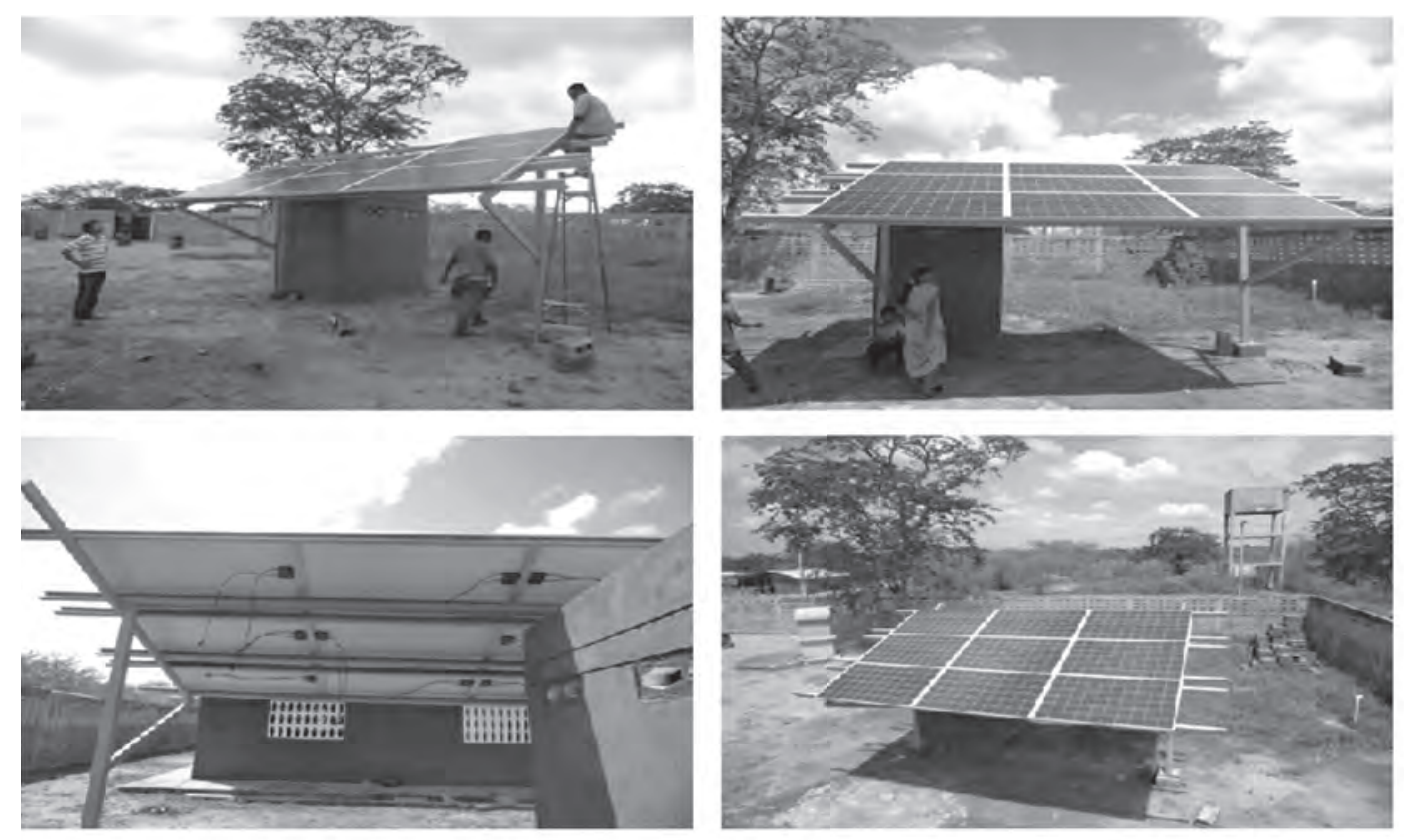

Figura 3. Instalación del sistema fotovoltaico en la comunidad Indígena Etkojo >ole.

Monitoreo de calidad de agua: Los datos promedio de los parámetros fisicoquímicos y bacteriológicos medidos en los puntos seleccionados de la comunidad, se presentan en la tabla 2. Los valores obtenidos para cada parámetro en la totalidad de las muestras están dentro de los límites máximos permisibles, establecidos en la resolución 2115 de 2007 (MPS \& MAVDT, 2007). Se encontraron diferencias significativas en algunas variables fisicoquímicas respecto al punto de muestreo, que fueron: oxígeno disuelto $(p=0,015)$, dureza total $(p=0,012)$, sólidos disueltos totales $(p=0,033)$, turbiedad $(p=0,025)$ y cloruro $(p=0,025)$. Las variaciones de oxígeno disuelto fueron menores en el pozo, registrando, en 
promedio, concentraciones de 3,27 \pm 0,22mg/L, aumentando a través del recorrido, lo que indica, que el agua sufría modificaciones en su calidad, a través de su recorrido, desde la captación hasta las viviendas
Los niveles de dureza no presentan sabores indeseables ni requieren tratamientos adicionales para remover excesos de dureza. Los SDT resultaron dentro de los rangos normales en la totalidad de los muestreos; la Agencia de Protección Ambiental (USEPA) sugiere un máximo de 500mg/L en agua potable y su variabilidad podría estar influenciada por la dureza (RIPDA, 2003).

Tabla 2. Resultados de las variables fisicoquímicas y microbiológicas, medidas en tres estaciones de la comunidad indígena Wayuu de Etkojo > ole, durante el periodo de muestreo.

\begin{tabular}{|c|c|c|c|c|c|c|}
\hline \multirow{2}{*}{ PARÁMETROS } & \multirow{2}{*}{ UNIDAD } & \multirow{2}{*}{$\mathbf{n}$} & \multicolumn{3}{|c|}{ PUINTOS DE MONITOREO* } & \multirow{2}{*}{$\begin{array}{c}\text { MÁXIMO } \\
\text { PERMISIBLE } \\
\text { Res } \\
\text { 2115/2007 }\end{array}$} \\
\hline & & & $\mathrm{P}$ & A & C & \\
\hline TEMPERATURA & ${ }^{\circ} \mathrm{C}$ & 4 & $28,7 \pm 0,5$ & $30,18 \pm 1,10$ & $27,63 \pm 0,1$ & N.R \\
\hline $\mathrm{pH}$ & Ad & 4 & $7,83 \pm 0,11$ & $8,23 \pm 0,17$ & $8,23 \pm 0,29$ & $6,5-9$ \\
\hline OXÍGENO DISUELTO & $\mathrm{mg} / \mathrm{L}$ & 4 & $3,27 \pm 0,22$ & $6,09 \pm 0,64$ & $6,89 \pm 0,49$ & N.R \\
\hline SALINIDAD & $\%$ & 4 & $0,10 \pm 0$ & $0,10 \pm 0$ & $0,10 \pm 0$ & N.R \\
\hline CONDUCTIVIDAD & $\mu \mathrm{s} / \mathrm{cm}$ & 4 & $734,5 \pm 14,3$ & $763,25 \pm 29,2$ & $749,75 \pm 16,8$ & $1000 \mu \mathrm{s} / \mathrm{cm}$ \\
\hline SATURACION DE OD & $\%$ & 4 & $46,83 \pm 3,07$ & $77,18 \pm 3,19$ & $81,23 \pm 3,60$ & N.R \\
\hline DUREZA TOTAL & $\mathrm{mg} / \mathrm{L} \mathrm{CaCO}_{3}$ & 4 & $169 \pm 10,16$ & $185,5 \pm 13,71$ & $124,4 \pm 17,45$ & $300 \mathrm{mg} / \mathrm{L}$ \\
\hline TURBIEDAD & UNT & 4 & $0,77 \pm 0,15$ & $1,37 \pm 0,44$ & $1,01 \pm 0,17$ & 2 UNT \\
\hline COLOR APARENTE & UPC & 4 & $5 \pm 0$ & $7,5 \pm 5$ & $5 \pm 0$ & 15 UPC \\
\hline ALCALINIDAD TOTAL & $\mathrm{mg} / \mathrm{L}$ & 4 & $164,4 \pm 32,3$ & $143,67 \pm 5,91$ & $174,35 \pm 8,85$ & $200 \mathrm{mg} / \mathrm{L}$ \\
\hline SOLIDOS DISUELTOS TOTALES & $\mathrm{mg} / \mathrm{L}$ & 4 & $53,75 \pm 2,9$ & $76,5 \pm 14,64$ & $61,25 \pm 7,41$ & N.R \\
\hline AMONIO & $\mathrm{mg} / \mathrm{L}$ & 4 & $0,05 \pm 0$ & $0,07 \pm 0$ & $0,06 \pm 0,02$ & N.R \\
\hline FOSFATO & $\mathrm{mg} / \mathrm{L}$ & 4 & $0,13 \pm 0$ & $0,14 \pm 0$ & $0,15 \pm 0,06$ & $0,5 \mathrm{mg} / \mathrm{L}$ \\
\hline SULFATO & $\mathrm{mg} / \mathrm{L}$ & 4 & $210,9 \pm 10,5$ & $199,2 \pm 29,42$ & $212,8 \pm 15,10$ & $250 \mathrm{mg} / \mathrm{L}$ \\
\hline CLORURO & $\mathrm{mg} / \mathrm{L}$ & 4 & $92,78 \pm 2,0$ & $49,57 \pm 2,67$ & $53,33 \pm 9,71$ & $250 \mathrm{mg} / \mathrm{L}$ \\
\hline NITRITO & $\mathrm{mg} / \mathrm{L}$ & 4 & 0 & 0 & $0,04 \pm 0,01$ & $0,1 \mathrm{mg} / \mathrm{L}$ \\
\hline NITRATO & $\mathrm{mg} / \mathrm{L}$ & 4 & $2,94 \pm 0,55$ & $2,60 \pm 0,42$ & $2,77 \pm 0,57$ & $10 \mathrm{mg} / \mathrm{L}$ \\
\hline HIERRO & $\mathrm{mg} / \mathrm{L}$ & 4 & $0,16 \pm 0$ & $0,22 \pm 0$ & $0,17 \pm 0,04$ & $0,3 \mathrm{mg} / \mathrm{L}$ \\
\hline ZINC & $\mathrm{mg} / \mathrm{L}$ & 4 & $1,05 \pm 0,25$ & $0,99 \pm 0,25$ & $0,99 \pm 0,25$ & $3 \mathrm{mg} / \mathrm{L}$ \\
\hline MANGANESO & $\mathrm{mg} / \mathrm{L}$ & 4 & 0 & 0 & $0,04 \pm 0,02$ & $0,1 \mathrm{mg} / \mathrm{L}$ \\
\hline CALCIO & $\mathrm{mg} / \mathrm{L}$ & 4 & $40 \pm 4,1$ & $33,75 \pm 4,79$ & $42,50 \pm 2,89$ & $60 \mathrm{mg} / \mathrm{L}$ \\
\hline COLIFORMES FECALES & UFC & 4 & 0 & 0 & 0 & N.R \\
\hline COLIFORMES TOTALES & UFC & 4 & 0 & $7,50 \pm 15$ & 0 & 0 UFC \\
\hline IRCA & ------ & - & 0 & $7,21 \pm 14,42$ & 0 & -------- \\
\hline
\end{tabular}

n: Numero de Muestras *Puntos de Monitoreo; P: Pozo; A: Alberca-Depósito de Almacenamiento; C: Casa. N.R. No existen rangos en la de referencia. 
El análisis de calidad del agua de consumo, mediante el IRCA, calificó el nivel de riesgo, con un valor de cero (0), excepto en el depósito de almacenamiento colectivo durante el primer monitoreo, con un valor de 7,21, que significa nivel de riesgo bajo, originado por la presencia de coliformes totales, debido a los días transcurridos desde la última limpieza y, muchas veces, falta cuidado para mantener el depósito cerrado; sin embargo, no se encontraron coliformes fecales.

La calidad del agua no se vio afectada, desde el punto de vista fisicoquímico, por contaminación biológica con coliformes fecales (excepto lo antes mencionado), se puede afirmar que el agua es aceptada para consumo. Los niveles de turbidez fueron muy bajos (menores a 2 UNT), lo cual, es favorable para la calidad del agua, debido a que los altos niveles de turbiedad pueden estar asociados con presencia de microorganismos (APHA et al. 2005). INESAG (2013) registró para la comunidad Etkojo>ole concentraciones promedio de 447,4 UFC/100mL, de coliformes totales, determinadas en el depósito de almacenamiento colectivo, condicionadas por el periodo de lluvia durante los muestreos; la disposición de excretas, próximos a la fuente de agua y malos hábitos de higiene, en contraposición a los datos registrados, los cuales, fueron escasos, debido a las condiciones de sequía durante el periodo de muestreo y las mejoras en hábitos de higiene.

Formación de la comunidad en los campos de acción: Se registró una asistencia promedio de 17 mujeres, 9 hombres y 4 adultos mayores, pertenecientes a las 22 familias de la comunidad indígena y 120 niños integrantes del Centro Etnoeducativo, para un total de 150 personas, capacitadas en las temáticas antes descritas. La participación, la apropiación y la interacción de la comunidad, propició los compromisos en manejo y control de hábitos higiénicos, ahorro y uso eficiente del agua, subsistencia alimentaria e inspección y mantenimiento del sistema fotovoltaico. Un adecuado aprovechamiento del agua, así como la incorporación o mejoras de los hábitos de higiene en la comunidad, mediante las capacitaciones, han producido cambios en el rol doméstico y han ocasionado la disminución de los contaminantes biológicos del agua. Toda esta panorámica refleja que el conocimiento, es decir, la forma como los seres humanos perciben, entienden y organizan sus respuestas al entorno, está condicionado por la cultura (Rodríguez, 2008).

Subsistencia alimentaria: Se reportaron diferentes resultados por cada método de riego empleado, teniendo en cuenta las condiciones de las plantas respecto a su crecimiento y desarrollo, lo cual, depende de la eficiencia de conducción y de distribución del agua en la parcela demostrativa, como se presenta en la tabla 3. Los resultados indicaron que la técni- ca de riego viable, para ser utilizada en esta zona, es el riego por goteo solar Kondenskompressor; el sistema fue efectivo en lo que respecta al control y a la demanda del agua. Con este sistema, los cultivos obtuvieron productividad promedio del $60 \%$ del total sembrado.

Los métodos de riego operaron bajo el uso del sistema fotovoltaico para la extracción del agua del pozo y el riego por goteo solar representó una alternativa para el ahorro de agua, siendo eficiente para enfrentar, adaptarse y mitigar los efectos del cambio climático. Para los objetivos de mitigación y adaptación, los sistemas de goteo artesanales generan mejores resultados (Zou et al. 2013).

El uso de herramientas participativas permite involucrar a las comunidades en la toma de decisiones, generando procesos organizacionales y de control, con conocimientos proporcionados, para llevar a cabo las actividades acordes a su entorno. El sistema fotovoltaico y la adecuación de los sistemas de distribución y de almacenamiento de agua, garantizaron la disponibilidad del recurso, como una medida de adaptación al cambio climático, lo cual, sirvió de incentivo para que la comunidad se apropiara rápidamente de la tecnología.

Los valores promedio de los parámetros medidos para la determinación de la calidad de agua para consumo humano, se encuentran dentro del rango permisible para los estándares nacionales e internacionales; sin embargo, el riesgo inminente de contaminación está latente si no se consideran o conservan los hábitos de higiene y de manipulación del recurso.

Los métodos de riego revelaron la rentabilidad del ahorro de agua para enfrentar, adaptarse y mitigar el cambio climático y tiene beneficios para el desarrollo económico sostenible. Además, el estudio sugiere que el riego por goteo solar es el que mejor funciona en pro de alcanzar los objetivos de mitigación y adaptación, acompañado del bombeo fotovoltaico, como un elemento vital para mejorar la producción rural y la seguridad alimentaria.

Agradecimientos: Al centro de investigaciones y el Instituto de aprovechamiento de Agua de la Universidad de La Guajira. Conflictos de interés: El manuscrito fue preparado y revisado con la participación de todos los autores, quienes declaramos que no existe ningún conflicto de interés que ponga en riesgo la validez de los resultados presentados. Financiación: Este estudio fue financiado por Colciencias, a través del proyecto ideas para el cambio-soluciones en agua y pobreza. 
Tabla 3. Evaluación de los sistemas de Riego para los cultivos implementados en la parcela demostrativa.

\begin{tabular}{|c|c|c|c|c|c|c|}
\hline \multirow{2}{*}{$\begin{array}{c}\text { Tipos de cultivo } \\
\begin{array}{c}\text { Nombre de la } \\
\text { planta }\end{array}\end{array}$} & \multirow{2}{*}{$\begin{array}{c}\text { Tiempo de } \\
\text { evaluación } \\
\text { Días }\end{array}$} & \multirow{2}{*}{$\begin{array}{l}\text { Criterios de } \\
\text { Evaluación }\end{array}$} & \multicolumn{4}{|c|}{ Sistema de Riego } \\
\hline & & & $\begin{array}{l}\text { Regadera } \\
\text { manual }\end{array}$ & $\begin{array}{l}\text { Riego por } \\
\text { olla porosa }\end{array}$ & $\begin{array}{l}\text { Riego por } \\
\text { goteo con } \\
\text { manguera }\end{array}$ & $\begin{array}{l}\text { Riego por goteo solar } \\
\text { Kondenskompressor }\end{array}$ \\
\hline \multirow{5}{*}{$\begin{array}{c}\text { Lycopersicum } \\
\text { esculentum } \\
\text { Nombre Común: } \\
\text { Tomate }\end{array}$} & \multirow{5}{*}{60} & $A C$ & $\mathrm{E}$ & $\mathrm{E}$ & $\mathrm{E}$ & E \\
\hline & & DA & $10 \%$ & $25 \%$ & $90 \%$ & $90 \%$ \\
\hline & & $\mathrm{COA}$ & A & $\mathrm{B}$ & $M$ & $\mathrm{~B}$ \\
\hline & & CA & $E$ & $\mathrm{E}$ & $E$ & $E$ \\
\hline & & $\mathrm{CP}$ & $9 \%$ & $22 \%$ & $31 \%$ & $44 \%$ \\
\hline \multirow{5}{*}{$\begin{array}{l}\text { Capsicum } \\
\text { annuum } \\
\text { Nombre Común: } \\
\text { Pimentón }\end{array}$} & \multirow{5}{*}{60} & $A C$ & $\mathrm{E}$ & E & $\mathrm{E}$ & $\mathrm{E}$ \\
\hline & & DA & $10 \%$ & $25 \%$ & $90 \%$ & $90 \%$ \\
\hline & & $\mathrm{COA}$ & A & $\mathrm{B}$ & $M$ & B \\
\hline & & CA & $\mathrm{E}$ & $\mathrm{E}$ & $\mathrm{E}$ & $\mathrm{E}$ \\
\hline & & $\mathrm{CP}$ & $15 \%$ & $34 \%$ & $39 \%$ & $52 \%$ \\
\hline \multirow{5}{*}{$\begin{array}{c}\text { Phaseolus } \\
\text { vulgaris } \\
\text { Nombre Común: } \\
\text { Frijol Tinto o } \\
\text { cabecita negra }\end{array}$} & \multirow{5}{*}{90} & $\mathrm{AC}$ & ME & ME & ME & ME \\
\hline & & DA & $10 \%$ & $25 \%$ & $90 \%$ & $95 \%$ \\
\hline & & $\mathrm{COA}$ & A & B & $M$ & $\mathrm{~B}$ \\
\hline & & CA & $\mathrm{E}$ & $\mathrm{E}$ & $\mathrm{E}$ & $\mathrm{E}$ \\
\hline & & $\mathrm{CP}$ & $20 \%$ & $52 \%$ & $51 \%$ & $80 \%$ \\
\hline \multirow{5}{*}{$\begin{array}{c}\text { Coriandrum } \\
\text { sativum } \\
\text { Nombre Común: } \\
\text { Cilantro }\end{array}$} & \multirow{5}{*}{60} & $A C$ & $\mathrm{E}$ & $\mathrm{E}$ & $\mathrm{E}$ & $\mathrm{E}$ \\
\hline & & DA & $10 \%$ & $25 \%$ & $90 \%$ & $90 \%$ \\
\hline & & $\mathrm{COA}$ & A & $\mathrm{B}$ & $M$ & $\mathrm{~B}$ \\
\hline & & CA & $\mathrm{E}$ & E & $E$ & $E$ \\
\hline & & $\mathrm{CP}$ & $10 \%$ & $45 \%$ & $50 \%$ & $64 \%$ \\
\hline
\end{tabular}

AC: Adaptación a los cultivos, DA: Demanda de agua, COA: Consumo de agua, CA: Control de agua, CP: Crecimiento de la planta, Valoración ME: Muy Eficiente, E: Eficiente, D: Deficiente, Consumo de agua, A: Alto, M: Medio, B: Bajo, Rango de Eficiencia, Riego Tradicional 10 - 30\% Riego por Goteo 90-95\% (FAO, 2008).

\section{BIBLIOGRAFÍA}

1. AMERICAN PUBLIC HEALTH ASSOCIATION (APHA). AMERICAN WATER WORKS ASSOCIATION (AWWA). WATER ENVIRONMENT FEDERATION (WEF). 2005. Standard Methods for the Examination of Water and Wastewater. (USA). $21^{\text {st }}$ edition.1368p.

2. AUBEL, J. 2000. Manual de evaluación participativa del programa. Catholic Relief Services-Child Survival Technical Support-USAID. (USA).104p.

3. CAMARE, H.; LANE, D. 2015. Adaptation analysis for environmental change in coastal communities, Socio-Economic Planning Sciences. (UnitedKingdom). 51:34-45.
4. CHAMBERS, R. 1994.The origins and practice of Participatory Rural Appraisal, World Development. (UnitedKingdom). 22(7):953-969.

5. CORPORACIÓN AUTÓNOMA REGIONAL DE LA GUAJIRA -CORPOGUAJIRA-. 2009. Plan de Gestión Ambiental Regional PGAR 2009-2019. Riohacha. (Colombia). 226p.

6. DEPARTAMENTO ADMINISTRATIVO NACIONAL DE ESTADÍSTICA -DANE-. 2005. Censo General 2005 Nivel Nacional. Disponible desde Internet en: www. dane.gov.co/files/libroCenso2005nacional.pdf (con acceso el 03/03/2015). 
7. ECHEVERRI, J. 2009. Pueblos indígenas y cambio climático: el caso de la Amazonía colombiana. Bol. Inst. Francés Estudios Andinos. (Francia). 38(1):1328.

8. FAO. ORGANIZACIÓN DE LAS NACIONES UINIDAS PARA LA AGRICULTURA Y LAS ALIMENTACIÓN. OFICINA REGIONAL PARA AMÉRICA LATINA Y EL CARIBE. 2008. El desarrollo del microriego en América Central. Oportunidades, Limitaciones y Desafíos. (Chile). 88p.

9. FELDT, H. 2011. Pueblos indígenas y cambio climático. Relación entre cambio climático y pueblos indígenas y sus posiciones en el contexto de las negociaciones en la Convención Marco sobre el Cambio Climático. Programa "Fortalecimiento de Organizaciones Indígenas en América Latina, PROINDIGENA". Ed. Deutsche Gesellschaft für Internationale Zusammenarbeit. (Chile) 18p.

10. FORERO, E.; HERNÁNDEZ, Y.; ZAFRA, C. 2014. Percepción latinoamericana de cambio climático: Metodologías, herramientas y estrategias de adaptación en comunidades locales. Una revisión. Rev. U.D.C.A Act. \& Div. Cient. (Colombia). 17(1):73-85.

11. GOBERNACIÓN DE LA GUAJIRA. 2016. Plan de desarrollo del Departamento de La Guajira 2016 - 2019 Oportunidad para Todos y Propósito de País. Disponible desde Internet en: http://www.laguajira.gov. co (con acceso el 05/01/2016).

12. HALL, G.; PATRINOS, H. 2010. Indigenous Peoples, Poverty and Development. Ed. World Bank. (USA). 339p.

13. INTERGOVERNMENTAL PANEL ON CLIMATE CHANGE -IPCC-. 2014. Climate Change. Impacts, Adaptation, and Vulnerability. Part A: Global and Sectoral Aspects. Working Group II Contribution to the Fifth Assessment Report of the Intergovernmental Panel on Climate Change. Ed. Cambridge University Press. (USA). 1150p.

14. INSTITUTO DE ESTUDIOS AMBIENTALES Y APROVECHAMIENTO DE AGUA -INESAG-. 2013. Evaluación de la calidad del agua para consumo humano y doméstico en la zona rural del municipio de Manaure, La Guajira. (Colombia). 180p.

15. KRONIK, J.; VERNER, D. 2010. Indigenous Peoples and Climate Change in Latin America and the Caribbean.
The International Bank for Reconstruction and Development. Ed. World Bank. (USA). 121p.

16. MELKONYAN, A. 2015. Climate change impact on water resources and crop production in Armenia. Agr. Water Managem. (Netherlands). 161:86-101.

17. MINISTERIO DE AMBIENTE Y DESARROLLO SOSTENIBLE DE LA REPUBLICA DE COLOMBIA MADS-. 2013. Adaptación basada en Comunidades - AbC. 64p.

18. MINISTERIO DE AMBIENTE VIVIENDA Y DESARROLLO TERRITORIAL -MAVDT-. 2010. Plan Departamental para el Manejo Empresarial de los Servicios de Agua y Saneamiento La Guajira. República de Colombia, Viceministerio de Agua y Saneamiento.112p.

19. MINISTERIO DE LA PROTECCIÓN SOCIAL -MPS-; MINISTERIO DE AMBIENTE, VIVIENDA Y DESARROLLO TERRITORIAL -MAVDT-. 2007. Resolución 2115 de 2007. Por medio de la cual se señalan características, instrumentos básicos y frecuencias del sistema de control y vigilancia para la calidad del agua para consumo humano. 23p.

20. MUSA. PROYECTO PARA MODELOS URBANOS Y RURALES EN SALUD AMBIENTAL. 2002. Educación sanitaria: Marco General y Metodología para el Diagnostico de Prácticas de Higiene Actuales Relacionados con Agua y Saneamiento. Módulo de Capacitación. (Argentina). 21p.

21. ORGANIZACIÓN MUINDIAL DE LA SALUD -OMS-; OFICINA DEL ALTO COMISIONADO DE LAS NACIONES UNIDAS PARA LOS DERECHOS HUMANOS -ACNUDH-; PROGRAMA DE LAS NACIONES UNIDAS PARA LOS ASENTAMIENTOS HUMANOS - ONU-. 2010. The Right to Water, Fact Sheet No. 35. 2010. Disponible desde Internet en: www.ohchr.org/Documents/Publications/FactSheet35en.pdf (con acceso el 05/11/2015).

22. PÉREZ, C.; NICKLIN, C.; DANGLES, O.; VANEK, S.; HALLOY, S.; GARRETT, K.; FORBES, G. 2010. Climate change in the high Andes: Implications and adaptation strategies for small-scale farmers. Int. J. Environ. Cult. Econ. Soc. Sustain. (Australia). 6(5):71-88.

23. PETZOLD, J.; BEATE, R. 2015. Climate change adaptation under a social capital approach e ananalytical framework for small islands. Ocean \& Coastal Management. (Northern Ireland). 112:36-43. 
24. RED IBEROAMERICANA DE POTABILIZACIÓN Y DEPURACIÓN DEL AGUA -RIPDA-. 2003. Agua potable para comunidades rurales, reúso y tratamientos avanzados de aguas residuales domésticas. (Argentina). 269p.

25. RODRÍGUEZ, L. 2008. Factores Sociales y Culturales Determinantes en Salud: La Cultura como una Fuerza para Incidir en Cambios en Políticas de Salud Sexual y Reproductiva. (Argentina). 21p.

26. SARZYNSKI, A. 2015. Public participation, civic capacity, and climate change adaptation in cities, Urban Climate. Disponible desde Internet en: http://dx.doi. org/10.1016/j.uclim.2015.08.002 (con acceso el 01/11/2015).

27. SOLÍS, R.; SALVATIERRA, B. 2013. Percepción social del cambio climático en Áreas Destinadas Voluntariamente a la Conservación en comunidades indígenas de Oaxaca y Chiapas. Temas Antropológicos. Rev. Cient. Inv. Reg. (México) 35(1):29-53.

28. ULLOA, A.; ESCOBAR, E.; DONATO, L.; ESCOBAR, P. 2008. Mujeres indígenas y cambio climático. Per- spectivas latinoamericanas. Ed. UNAL-Fundación Natura-UNODC. (Colombia). 234p.

29. VERNER, D. 2010. Reducing Poverty, Protecting Livelihoods and Building Assets in a Changing Climate: Social Implications of Climate Change in Latin America and the Caribbean. Ed. World Bank. Washington D.C. (USA). 460p.

30. ZHAO, A.; ZHU, X.; LIU, X.; PAN, Y.; ZUO, D. 2016. Impacts of land use change and climate variability on green and blue water resources in the Weihe River Basin of northwest China. Catena. (Netherlands). 137:318-327.

31. ZOU, X.; LI, Y.; CREMADES, R.; GAO, Q.; WAN, Y.; QIN, X. 2013. Cost-effectiveness analysis of water-saving irrigation technologies based on climate change response: A case study of China. Agr. WaterManagem. (Netherlands).129:9-20.

Recibido: Junio 8 de 2016

Aceptado: Febrero 9 de 2017

Cómo citar:

Galindo Montero, A.; Pérez Montiel, J.; Rojano Alvarado, R. 2017. Medidas de adaptación al cambio climático en una comunidad indígena del norte de Colombia. Rev. U.D.C.A Act. \& Div. Cient. 20(1): 187-197. 